\title{
ON FRACTIONAL SUPERSYMMETRIC QUANTUM MECHANICS: THE FRACTIONAL SUPERSYMMETRIC OSCILLATOR
}

\author{
M. DAOUD \\ Département de Physique \\ Faculté des Sciences \\ Université Ibnou Zohr \\ B.P. 28/S, Agadir, Morocco \\ M.R. KIBLER \\ Institut de Physique Nucléaire de Lyon \\ IN2P3-CNRS et Université Claude Bernard \\ 43, Boulevard du 11 Novembre 1918 \\ F-69622 Villeurbanne Cedex, France
}

\begin{abstract}
The Hamiltonian for a fractional supersymmetric oscillator is derived from three approaches. The first one is based on the $Q$-uon $\rightarrow$ boson $+k$-fermion decomposition. The second one starts from a generalized Weyl-Heisenberg algebra. Finally, the third one relies on the quantum algebra $U_{q}\left(s l_{2}\right)$ where $q$ is a root of unity.
\end{abstract}

\section{Generalities about supersymmetry}

What is supersymmetry? Roughly speaking SUperSYmmetry or SUSY can be defined as a symmetry between bosons and fermions (as considered as elementary particles or simply as degrees of freedom). In other words, SUSY is based on the postulated existence of operators $Q_{\alpha}$ which transform a bosonic field into a fermionic field and vice versa. In the context of quantum mechanics, such symmetry operators induce a $Z_{2}$-grading of the Hilbert space of quantum states. In a more general way, fractional SUSY corresponds to a $Z_{k}$-grading for which the Hilbert space involves both bosonic degrees of freedom (associated to bosons) and $k$-fermionic degrees of freedom (associated to $k$-fermions to be described below) with $k \in \mathbf{N} \backslash\{0,1\}$; the case $k=2$ corresponds to ordinary SUSY.

Where does SUSY come from ? The concept of SUSY goes back to the sixtees when many attemps were done in order to unify external symmetries (described by the Poincaré group) and internal symmetries (described by gauge groups) for elementary particles. These attempts led to a no-go theorem by Coleman and Mandula ${ }^{1}$ in 1967 which states that, under reasonable assumptions concerning the $S$-matrix, the unification of internal and external symmetries can be achieved solely through the introduction of the direct product of 
the Poincaré group with the relevant gauge group. In conclusion, this unification brings nothing new since it simply amounts to consider separately the two kinds of symmetries. A way to escape this no-go theorem was proposed by Haag, Lopuszanski and Sohnius ${ }^{2}$ in 1975: The remedy consists in replacing the Poincaré group by a Poincaré supergroup (or $Z_{2}$-graded Poincaré group).

Let us briefly discuss how to introduce the Poincaré supergroup. We know that the Poincaré group has ten generators ( $\operatorname{six} M_{\mu \nu} \equiv-M_{\nu \mu}$ and four $P_{\mu}$ with $\mu, \nu \in\{0,1,2,3\})$ : Three $M_{\mu \nu}$ describe ordinary rotations, three $M_{\mu \nu}$ describe special Lorentz transformations and the four $P_{\mu}$ describe space-time translations. The Lie algebra of the Poincaré group is characterized by the commutation relations

$$
[M, M]=M, \quad[M, P]=P, \quad[P, P]=0
$$

(written in a symbolic way). The minimal extension of the Poincaré group into a Poincaré supergroup gives rise to a Lie superalgebra (or $Z_{2}$-graded Lie algebra) involving the ten generators $M_{\mu \nu}$ and $P_{\mu}$ plus four new generators $Q_{\alpha}$ (with $\alpha \in\{1,2,3,4\}$ ) referred to as supercharges. The Lie superalgebra of the Poincaré supergroup is then described by the commutation relations

$$
\begin{gathered}
{[M, M]=M, \quad[M, P]=P, \quad[P, P]=0} \\
{[Q, M]=Q, \quad[Q, P]=0}
\end{gathered}
$$

and the anticommutation relations

$$
\{Q, Q\}=0
$$

(in a symbolic way).

What is the consequence of this increase of symmetries (i.e., passing from 10 to 14 generators) and of the introduction of anticommutators? In general, an increase of symmetry yields an increase of degeneracies. For instance, in condensed matter physics, passing from the tetragonal symmetry to the cubical symmetry leads, from a situation where the degrees of degeneracy are 1 and 2, to a situation where the degrees of degeneracy are 1,2 and 3 (in the absence of accidental degeneracies). Another possible consequence of the increase of symmetry is the occurrence of new states or new particles. For instance, the number of particles is doubled when going from the Schrödinger equation (with Galilean invariance) to the Dirac equation (with Lorentz invariance): To each particle of mass $m$, spin $S$ and charge $e$ is associated an antiparticle of mass $m$, spin $S$ and charge $-e$. In a similar way, when passing from the Poincaré group to the Poincaré supergroup, we associate to a known particle of mass $m$, 
spin $S$ and charge $e$ a new particle of mass $m^{\prime}$, spin $S^{\prime}=\left|S \pm \frac{1}{2}\right|$ and charge $e$. (We have $m^{\prime} \neq m$ because supersymmetry is a broken symmetry.) The new particle is called a sparticle or a particlino according to whether as the known particle is a fermion or a boson. In terms of field theory, the sparticle or particlino field results from the action of a supercharge $Q_{\alpha}$ on the particle field (and vice versa):

$$
\begin{gathered}
Q_{\alpha}: \text { fermion } \mapsto \text { sfermion }=\text { boson } \\
Q_{\alpha}: \text { boson } \mapsto \text { bosino }=\text { fermion }
\end{gathered}
$$

(see also Ref. 3). We thus speak of a selectron (a particle of spin 0 associated to the electron) and of a photino (a particle of spin $\frac{1}{2}$ associated to the photon).

It is not our intention to further discuss SUSY from the viewpoint of elementary particles. We shall rather focus our attention on some aspects of fractional SUSY and, more precisely, on fractional supersymmetric or parasupersymmetric (one-dimensional) nonrelativistic quantum mechanics, in the vein of the works in Refs. 4-23. From the point of view of quantum mechanics, a SUSY Hamiltonian comprises both bosonic and fermionic degrees of freedom. Then, the problem is to associate a SUSY Hamiltonian to the Hamiltonian of a given dynamical system. Here, we are interested in one of the simplest dynamical systems, namely, the harmonic oscillator. We know that the ordinary oscillator can be described in terms of boson operators. We shall see that the SUSY oscillator can be described in terms of boson and fermion operators (reflecting a $Z_{2}$-grading) and, more generally, that the fractional SUSY oscillator can be described in terms of boson and $k$-fermion operators (corresponding to a $Z_{k}$-grading with $\left.k \in \mathbf{N} \backslash\{0,1\}\right)$. The rest of this paper is thus devoted to the construction (via three methods) of the Hamiltonian for a fractional supersymmetric (or SUSY) oscillator.

This school was dedicated to the memory of Louis Michel. One of the authors (M.R. K.) has had the chance to meet Louis Michel at the Université de Montréal in 1976. Louis will remain an example for him and for many of us. We will all remember the exceptional qualities of the man as a scientist, as a private person, as a public figure and as a teacher.

\section{A quon approach to the SUSY oscillator}

\subsection{The $Q$-uon $\rightarrow$ boson $+k$-fermion decomposition}

We shall limit ourselves to give an outline of this decomposition (see Dunne et al. ${ }^{24}$ and Mansour et al. ${ }^{25}$ for a more rigorous mathematical presentation based on the isomorphism between the braided $Z$-line and the $(z, \theta)$ superspace). We start from a $Q$-uon algebra spanned by three operators $a_{-}$, 
$a_{+}$and $N$ satisfying the relationships ${ }^{26}$

$$
\begin{gathered}
a_{-} a_{+}-Q a_{+} a_{-}=1 \\
N a_{-}-a_{-} N=-a_{-}, \quad N a_{+}-a_{+} N=+a_{+}
\end{gathered}
$$

where $Q$ is generic (a real number different from zero). Let us consider the $Q$-deformed coherent state ${ }^{27}$

$$
\mid Z):=\sum_{n=0}^{\infty} \frac{\left(Z a_{+}\right)^{n}}{[n]_{Q} !}|0\rangle=\sum_{n=0}^{\infty} \frac{Z^{n}}{\left([n]_{Q} !\right)^{\frac{1}{2}}}|n\rangle
$$

(with $|n\rangle$ such that $N|n\rangle=n|n\rangle$ and $Z \in \mathbf{C}$ ) where

$$
[n]_{Q}:=\frac{1-Q^{n}}{1-Q}
$$

and

$$
[n]_{Q} !:=[1]_{Q}[2]_{Q} \cdots[n]_{Q} \quad \text { for } n \in \mathbf{N}^{*} \text { and }[0]_{Q} !:=1
$$

If we assume that

$$
Q \rightarrow q:=\exp \left(\frac{2 \pi \mathrm{i}}{k}\right), \quad k \in \mathbf{N} \backslash\{0,1\}
$$

then we have $[k]_{Q} ! \rightarrow[k]_{q} !=0$. Therefore, in order to give a sense to $\left.\mid Z\right)$ for $Q \rightarrow q$, we have to do the replacement

$$
a_{+} \leadsto f_{+} \text {with }\left(f_{+}\right)^{k}=0
$$

We thus end up with what we call a $k$-fermionic algebra spanned by the operators $f_{-}, f_{+}$and $N$ completed by the adjoints $f_{+}^{+}$and $f_{-}^{+}$of $f_{+}$and $f_{-}$, respectively. ${ }^{20-22}$ The defining relations for the $k$-fermionic algebra are

$$
\begin{gathered}
f_{-} f_{+}-q f_{+} f_{-}=1 \\
N f_{-}-f_{-} N=-f_{-}, \quad N f_{+}-f_{+} N=+f_{+} \\
\left(f_{-}\right)^{k}=\left(f_{+}\right)^{k}=0
\end{gathered}
$$

and similar relations for $f_{+}^{+}$and $f_{-}^{+}$. The case $k=2$ corresponds to ordinary fermion operators and the case $k \rightarrow \infty$ to ordinary boson operators. The $k$ fermions are objects interpolating between fermions and bosons. They share 
some properties with the para-fermions ${ }^{4-6}$ and the anyons as introduced by Goldin et al. ${ }^{28}$ (see also Ref. 29). If we define

$$
b_{ \pm}:=\lim _{Q \rightarrow q} \frac{\left(a_{ \pm}\right)^{k}}{[k]_{Q} !}
$$

we obtain

$$
b_{-} b_{+}-b_{+} b_{-}=1
$$

so that the operators $b_{-}$and $b_{+}$can be considered as ordinary boson operators. This is at the root of the two following results. ${ }^{20}$

As a first result, the set $\left\{a_{-}, a_{+}\right\}$gives rise, for $Q \rightarrow q$, to two commuting sets: The set $\left\{b_{-}, b_{+}\right\}$of boson operators and the set of $k$-fermion operators $\left\{f_{-}, f_{+}\right\}$. As a second result, this decomposition leads to the replacement of the $Q$-deformed coherent state $\mid Z$ ) by the so-called fractional supercoherent state

$$
\mid z, \theta):=\sum_{r=0}^{\infty} \sum_{s=0}^{k-1} \frac{\theta^{s}}{\left([s]_{q} !\right)^{\frac{1}{2}}} \frac{z^{r}}{\sqrt{r !}}|k r+s\rangle
$$

where $z$ is a (bosonic) complex variable and $\theta$ a ( $k$-fermionic) generalized Grassmann variable ${ }^{4,6,30,31}$ with $(\theta)^{k}=0$. The fractional supercoherent state $\left.\mid z^{k}, \theta\right)$ can be seen to be a linear combination of the coherent states introduced by Vourdas ${ }^{32}$ with coefficients in the generalized Grassmann algebra spanned by $\theta$ and the derivative $\partial_{\theta}$.

In the case $k=2$, the fractional supercoherent state $\mid z, \theta)$ turns out to be a coherent state for the ordinary (or $Z_{2}$-graded) supersymmetric oscillator. ${ }^{33} \mathrm{We}$ construct below a fractional (or $Z_{k}$-graded) supersymmetric oscillator which can be associated to the fractional supercoherent state $\mid z, \theta)$ with $k=3,4, \cdots$.

\subsection{A quon approach to the Weyl-Heisenberg algebra}

In this section, the basic ingredients consist of a pair of ordinary bosons $\left(b_{-}, b_{+}\right)$ and a pair of $k$-fermions $\left(f_{-}, f_{+}\right)$. The $f$ 's satisfy $q$-commutation relations and the $b$ 's usual commutation relations (see the relations above). In addition, the $f$ 's commute with the $b$ 's. Indeed, the two pairs $\left(b_{-}, b_{+}\right)$and $\left(f_{-}, f_{+}\right)$ may be considered as originating from a pair of $Q$-uons $\left(a_{-}, a_{+}\right)$through the above-described $Q$-uon $\rightarrow$ boson $+k$-fermion decomposition.

Let us define the two operators $X_{-}$and $X_{+}$by

$$
X_{-}:=b_{-}\left[f_{-}+\frac{\left(f_{+}\right)^{k-1}}{[k-1]_{q} !}\right], \quad X_{+}:=b_{+}\left[f_{-}+\frac{\left(f_{+}\right)^{k-1}}{[k-1]_{q} !}\right]^{k-1}
$$


and the operator $K$ by

$$
K:=f_{-} f_{+}-f_{+} f_{-}
$$

in terms of the operators $b_{-}, b_{+}, f_{-}$and $f_{+}$. Then, we can check that $X_{-}$, $X_{+}$and $K$ satisfy

$$
\begin{gathered}
X_{-} X_{+}-X_{+} X_{-}=1, \quad K^{k}=1 \\
K X_{+}-q X_{+} K=0, \quad K X_{-}-q^{-1} X_{-} K=0
\end{gathered}
$$

Furthermore, the operator

$$
M:=X_{+} X_{-}
$$

satisfies the following commutation relations

$$
M X_{-}-X_{-} M=-X_{-}, \quad M X_{+}-X_{+} M=+X_{+}, \quad M K-K M=0
$$

The operators $X_{-}, X_{+}, K$ and $M$ thus generate an extended Weyl-Heisenberg algebra.

\subsection{The resulting fractional supersymmetric oscillator}

In the spirit of the work by Rubakov and Spiridonov, ${ }^{4}$ we introduce the $k$ projection operators

$$
\Pi_{i}:=\frac{1}{k} \sum_{s=0}^{k-1} q^{s i} K^{s}, \quad i=0,1, \cdots, k-1
$$

for the cyclic group $Z_{k}$, the two supercharges

$$
Q_{-}:=X_{-}\left(1-\Pi_{k-1}\right), \quad Q_{+}:=X_{+}\left(1-\Pi_{0}\right)
$$

and the Hamiltonian $H$ defined through

$$
\left(Q_{-}\right)^{k-1} Q_{+}+\left(Q_{-}\right)^{k-2} Q_{+} Q_{-}+\cdots+Q_{+}\left(Q_{-}\right)^{k-1}=\left(Q_{-}\right)^{k-2} H
$$

It is then a simple matter of calculation to show that the supercharges $Q_{-}$and $Q_{+}$are nilpotent operators with

$$
\left(Q_{-}\right)^{k}=\left(Q_{+}\right)^{k}=0
$$

and that the Hamiltonian $H$ can be written as a bilinear form involving $X_{-} X_{+}$ and $\Pi_{0}, \Pi_{1}, \cdots, \Pi_{k-1}$ with

$$
\begin{aligned}
H=X_{-} X_{+} \Pi_{1} & +\sum_{\ell=2}^{k-1}\left(X_{+} X_{-}-\ell+1\right)\left(\Pi_{0}+\Pi_{1}+\cdots+\Pi_{k-\ell-1}\right) \\
& +\sum_{\ell=2}^{k-1} \ell\left(X_{-} X_{+}+\frac{\ell-1}{2}\right) \Pi_{\ell}+X_{+} X_{-}\left(1-\Pi_{k-1}\right)
\end{aligned}
$$


In addition, we have the commutation relations

$$
H Q_{ \pm}-Q_{ \pm} H=0
$$

and thus $Q_{-}$and $Q_{+}$can be regarded as constants of motion. Finally, it can be seen that $H$ may be associated to the fractional supercoherent state $\mid z, \theta)$ with $k \in \mathbf{N} \backslash\{0,1\}$.

\subsection{Examples}

Example 1. As a first example, we take $k=2$, i.e., $q=-1$. Then, we have

$$
X_{-}:=b_{-}\left(f_{-}+f_{+}\right), \quad X_{+}:=b_{+}\left(f_{-}+f_{+}\right)
$$

and

$$
K:=f_{-} f_{+}-f_{+} f_{-}
$$

where $\left(b_{-}, b_{+}\right)$are ordinary bosons and $\left(f_{-}, f_{+}\right)$ordinary fermions. The operators $X_{-}, X_{+}$and $K$ satisfy

$$
\begin{gathered}
X_{-} X_{+}-X_{+} X_{-}=1, \quad K^{2}=1 \\
K X_{+}+X_{+} K=0, \quad K X_{-}+X_{-} K=0
\end{gathered}
$$

which reflect bosonic and fermionic degrees of freedom. The projection operators

$$
\Pi_{0}:=\frac{1}{2}(1+K), \quad \Pi_{1}:=\frac{1}{2}(1-K)
$$

are here simple chirality operators and the supercharges

$$
Q_{-}:=X_{-} \Pi_{0}, \quad Q_{+}:=X_{+} \Pi_{1}
$$

have the property

$$
\left(Q_{-}\right)^{2}=\left(Q_{+}\right)^{2}=0
$$

The Hamiltonian $H$ assumes the form

$$
H=Q_{-} Q_{+}+Q_{+} Q_{-}
$$

which can be rewritten as

$$
H=X_{+} X_{-} \Pi_{0}+X_{-} X_{+} \Pi_{1}
$$

It is clear that $H$ commutes with $Q_{-}$and $Q_{+}$. In terms of boson and fermion operators, we have

$$
Q_{-}=f_{+} b_{-}, \quad Q_{+}=f_{-} b_{+}
$$


and

$$
H=b_{+} b_{-}+f_{+} f_{-}
$$

so that $H$ corresponds to the ordinary (or $Z_{2}$-graded) supersymmetric oscillator whose energy spectrum $E$ is (in a symbolic way)

$$
E=1 \oplus 2 \oplus 2 \oplus \cdots
$$

with equally spaced levels, the ground state being a singlet (denoted by 1) and all the excited states (viz., an infinite sequence) being doublets (denoted by 2 ). Finally, note that the fractional supercoherent state $\mid z, \theta)$ with $k=2$ is a coherent state for the Hamiltonian $H$ (see Ref. 33).

Example 2. We continue with $k=3$, i.e.,

$$
q=\exp \left(\frac{2 \pi \mathrm{i}}{3}\right)
$$

In this case, we take

$$
X_{-}:=b_{-}\left[f_{-}+\frac{\left(f_{+}\right)^{2}}{[2]_{q} !}\right], \quad X_{+}:=b_{+}\left[f_{-}+\frac{\left(f_{+}\right)^{2}}{[2]_{q} !}\right]^{2}
$$

and

$$
K:=f_{-} f_{+}-f_{+} f_{-}
$$

where $\left(b_{-}, b_{+}\right)$are ordinary bosons and $\left(f_{-}, f_{+}\right)$are 3 -fermions. We hence have

$$
\begin{gathered}
X_{-} X_{+}-X_{+} X_{-}=1, \quad K^{3}=1 \\
K X_{+}-q X_{+} K=0, \quad K X_{-}-q^{-1} X_{-} K=0
\end{gathered}
$$

Our general definitions can be specialized to

$$
\begin{aligned}
& \Pi_{0}:=\frac{1}{3}\left(1+q^{3} K+q^{3} K^{2}\right) \\
& \Pi_{1}:=\frac{1}{3}\left(1+q^{1} K+q^{2} K^{2}\right) \\
& \Pi_{2}:=\frac{1}{3}\left(1+q^{2} K+q^{1} K^{2}\right)
\end{aligned}
$$

for the projection operators and to

$$
Q_{-}:=X_{-}\left(\Pi_{0}+\Pi_{1}\right), \quad Q_{+}:=X_{+}\left(\Pi_{1}+\Pi_{2}\right)
$$


for the supercharges with the property

$$
\left(Q_{-}\right)^{3}=\left(Q_{+}\right)^{3}=0
$$

By introducing the Hamiltonian $H$ via

$$
\left(Q_{-}\right)^{2} Q_{+}+Q_{-} Q_{+} Q_{-}+Q_{+}\left(Q_{-}\right)^{2}=Q_{-} H
$$

we obtain

$$
H=\left(2 X_{+} X_{-}-1\right) \Pi_{0}+\left(2 X_{+} X_{-}+1\right) \Pi_{1}+\left(2 X_{+} X_{-}+3\right) \Pi_{2}
$$

which can be rewritten as

$$
H=2 b_{+} b_{-}-1+2(1-2 q) f_{+} f_{-}+2(1+2 q) f_{+} f_{-} f_{+} f_{-}
$$

in terms of boson and 3 -fermion operators. We can check that $H$ commutes with $Q_{-}$and $Q_{+}$. The energy spectrum of $H$ reads

$$
E=1 \oplus 2 \oplus 3 \oplus 3 \oplus \cdots
$$

It contains equally spaced levels with a nondegenerate ground state (denoted as 1), a doubly degenerate first excited state (denoted as 2) and an infinite sequence of triply degenerate excited states (denoted as 3 ).

\section{A generalized Weyl-Heisenberg approach to the SUSY oscillator}

This second approach starts directly from a generalized Weyl-Heisenberg algebra. Let this algebra be spanned by four operators $Y_{-}, Y_{+}, N$ and $K$ (the analogues of $X_{-}, X_{+}, M$ and $K$ in Section 2) with the relations

$$
\begin{aligned}
& Y_{-} Y_{+}-Y_{+} Y_{-}=\sum_{s=0}^{k-1} f_{s}(N) \Pi_{s}, \quad K^{k}=1 \\
& K Y_{+}-q Y_{+} K=0, \quad K Y_{-}-q^{-1} Y_{-} K=0
\end{aligned}
$$

and

$$
N Y_{-}-Y_{-} N=-Y_{-}, \quad N Y_{+}-Y_{+} N=+Y_{+}, \quad N K-K N=0
$$

where $f_{s}$ is an arbitrary (reasonable) function, $\Pi_{s}$ the projection operator defined in Section 2 and $q$ is the same root of unity as before. By defining the supercharges $Q_{-}$and $Q_{+}$and the Hamiltonian $H$ as in Section 2 (with $X_{ \pm}$ replaced by $Y_{ \pm}$), we obtain again that $Q_{-}$and $Q_{+}$are nilpotent operators of 
order $k$ which commute with $H$. Furthermore, we find that the operator $H$ can be expressed as

$$
\begin{aligned}
H & =\left[(k-1) Y_{+} Y_{-}-\sum_{\ell=1}^{k-2}(k-1-\ell) f_{\ell}(N-1)\right] \Pi_{0} \\
& +\left[(k-1) Y_{-} Y_{+}-\sum_{\ell=1}^{k-2}(k-1-\ell) f_{\ell}(N-\ell+1)\right] \Pi_{1} \\
& +\left[(k-1) Y_{-} Y_{+}+f_{1}(N+1)-\sum_{\ell=2}^{k-2}(k-1-\ell) f_{\ell}(N-\ell+2)\right] \Pi_{2} \\
& +\sum_{\ell=3}^{k-1}\left[(k-1) Y_{+} Y_{-}+\sum_{j=1}^{\ell} j f_{j}(N+\ell-j)\right] \Pi_{\ell}
\end{aligned}
$$

which generalizes the Hamiltonian derived in Section 2.

\section{An $U_{q}\left(s l_{2}\right)$ approach to the SUSY oscillator}

In this section, we shall briefly show how it is possible to define an Hamiltonian for a fractional supersymmetric oscillator in terms of the generators of the quantum algebra $U_{q}\left(s l_{2}\right)$ with $q$ a root of unity. Let $J_{-}, J_{+}, q^{J_{3}}$ and $q^{-J_{3}}$ be the generators of $U_{q}\left(s l_{2}\right)$. They satisfy the relationships

$$
\begin{gathered}
J_{+} J_{-}-J_{-} J_{+}=\frac{q^{2 J_{3}}-q^{-2 J_{3}}}{q-q^{-1}} \\
q^{J_{3}} J_{+} q^{-J_{3}}=q J_{+}, \quad q^{J_{3}} J_{-} q^{-J_{3}}=q^{-1} J_{-}
\end{gathered}
$$

where $q$ is the same root of unity as before. It is straightforward to prove that the operator

$$
C:=J_{-} J_{+}+\frac{q q^{2 J_{3}}+q^{-1} q^{-2 J_{3}}}{\left(q-q^{-1}\right)^{2}}
$$

is an invariant of $U_{q}\left(s l_{2}\right)$.

We now define the operator $K$ by

$$
K:=q^{J_{3}}
$$

and the projection operators $\Pi_{s}(s=1,2, \cdots, k-1)$ as functions of $K$ as in Section 2. The two supercharges $Q_{-}$and $Q_{+}$are defined here by

$$
Q_{-}:=J_{-}\left(1-\Pi_{k-1}\right), \quad Q_{+}:=J_{+}\left(1-\Pi_{0}\right)
$$


and the Hamiltonian $H$ is defined from the multilinear form involving $Q_{-}$and $Q_{+}$given in Section 2. As a final result, we obtain for $H$ the expression

$$
\begin{aligned}
H & =J_{-} J_{+} \Pi_{1}+J_{+} J_{-}\left(1-\Pi_{k-1}\right) \\
& +\sum_{m=2}^{k-1}\left[J_{+} J_{-}+\left(G\left(J_{3}-m+1\right)+G\left(J_{3}-m+2\right)+\cdots+G\left(J_{3}-1\right)\right)\right] \\
& \times\left(\Pi_{0}+\Pi_{1}+\cdots+\Pi_{k-m-1}\right) \\
& +\sum_{m=2}^{k-1}\left[m J_{-} J_{+}-\left((m-1) G\left(J_{3}+1\right)+(m-2) G\left(J_{3}+2\right)+\cdots\right.\right. \\
& \left.\left.+G\left(J_{3}+m-1\right)\right)\right] \Pi_{m}
\end{aligned}
$$

where $G$ is defined by

$$
G(X):=\frac{q^{2 X}-q^{-2 X}}{q-q^{-1}}
$$

The Hamiltonian $H$ is thus given by a formula involving $J_{3}$ (through the function $G$ ) and the product $J_{+} J_{-}$(or alternatively the operators $J_{3}$ and $C$ ).

\section{Concluding remarks}

We have derived three different Hamiltonians for a supersymmetric oscillator. At this point, it is worthwhile to introduce some links between the three derivations.

In the three derivations, the $Z_{k}$-grading is introduced via the same relation, i.e., $K^{k}=1$, although the introduction of $K$ is specific to each approach. The operator $K$ is: (i) a bilinear form of $k$-fermion operators in the quon approach (Section 2) originally developed in Ref. 22, (ii) a formal operator in the generalized Weyl-Heisenberg approach (Section 3), and (iii) a generator of the quantum algebra $U_{q}\left(s l_{2}\right)$ in the $U_{q}\left(s l_{2}\right)$ approach (Section 4). The $Z_{k}$-grading manifests itself in Section 2 through the introduction of bosonic degrees of freedom and $k$-fermionic degrees of freedom but this is not the case in Sections 3 and 4.

The connection between the approaches in Sections 2 and 3 is easy to describe: To pass from the generalized Weyl-Heisenberg approach to the quon approach, it is sufficient to take $f_{s}=1$ for $s=1,2, \cdots, k-1$. It is also to be noted that the generalized Weyl-Heisenberg approach covers some published works: (i) for $k=2$, our generalized Weyl-Heisenberg approach may be 
specialized to the cases where

$$
\sum_{s=0}^{1} f_{s}(N) \Pi_{s}=1
$$

and

$$
\sum_{s=0}^{1} f_{s}(N) \Pi_{s}=1+c K, \quad c \in \mathbf{C}
$$

treated by Plyushchay ${ }^{15}$ and (ii) for $k \in \mathbf{N} \backslash\{0,1\}$, by taking

$$
\sum_{s=0}^{k-1} f_{s}(N) \Pi_{s}=\sum_{s=0}^{k-1} c_{s} K^{s}, \quad c_{s} \in \mathbf{C}
$$

our generalized Weyl-Heisenberg approach may be found to be equivalent to the one developed by Quesne and Vansteenquite. ${ }^{19}$

Finally, note that the connection between the approach in Section 4 and the ones in Sections 2 and 3 still deserves to be explored. This will be the object of a forthcoming paper.

To close this paper, let us mention three domains where (fractional) supersymmetry play or might play an important rôle. First, in condensed matter physics, the interest of fractional supersymmetry rests on its application to the fractional quantum Hall effect and supraconductivity at critical temperature. Second, in nuclear physics, it seems that (ordinary) supersymmetry has been observed in nuclear spectroscopy: Supersymmetry could relate the structure of odd-odd nuclei to even-even and odd- $A$ systems. ${ }^{34}$ Third, in elementary particle physics, (ordinary) supersymmetry imposes some limits on the masss of the Higgs boson : The mass should be below $130 \mathrm{GeV} / c^{2}$ so that the "events" observed at LEP200 (CERN) in August 2000 around $115 \mathrm{GeV} / c^{2}$ are compatible with the hypotheses of supersymmetry in high energy physics. As a conclusion, supersymmetry is still a chapter of physics under construction.

\section{Acknowledgments}

The senior author (M.R. K.) is grateful to the Organizing Committee of the 6th International School of Theoretical Physics SSPCM'2000, and more specifically Prof. T. Lulek, for inviting him to deliver this lecture. He is also indebted to Profs. M. Bożejko, J.D. Louck and A. Vourdas for interesting comments. 


\section{References}

1. S. Coleman and J. Mandula, Phys. Rev. 159, 1251 (1967).

2. R. Haag, J.T. Lopuszanski and M. Sohnius, Nucl. Phys. B 88, 257 (1975).

3. P. Fayet, Phys. Lett. B 64, 159 (1976).

4. V.A. Rubakov and V.P. Spiridonov, Mod. Phys. Lett. A 3, 1337 (1988).

5. J. Beckers and N. Debergh, Mod. Phys. Lett. A 4, 1209 (1989) ; Nucl. Phys. B 340, 767 (1990).

6. A.T. Filippov, A.P. Isaev and A.B. Kurdikov, Mod. Phys. Lett. A 7, 2129 (1992) ; Int. J. Mod. Phys. A 8, 4973 (1993).

7. S. Durand, Mod. Phys. Lett. A 7, 2905 (1992) ; Phys. Lett. B 312, 115 (1993) ; Mod. Phys. Lett. B 8, 2323 (1993).

8. R. Kerner, J. Math. Phys. 33, 403 (1992).

9. A. Khare, J. Phys. A: Math. Gen. 25, L749 (1992) ; J. Math. Phys. 34, 1274 (1993).

10. N. Debergh, J. Math. Phys. 34, 1270 (1992) ; J. Phys. A: Math. Gen. 26, 7219 (1993) ; 27, L213 (1994).

11. J.L. Matheus-Valle and M.A. R.-Monteiro, Mod. Phys. Lett. A 7, 3023 (1992) ; Phys. Lett. B 300, 66 (1993) ; L.P. Collatto and J.L. MatheusValle, J. Math. Phys. 37, 6121 (1996).

12. N. Mohammedi, Mod. Phys. Lett. A 10, 1287 (1995).

13. D. Bonatsos, P. Kolokotronis and C. Daskaloyannis, Mod. Phys. Lett. A 10, 2197 (1995).

14. J.A. de Azcárraga and A.J. Macfarlane, J. Math. Phys. 37, 1115 (1996).

15. M.S. Plyushchay, Ann. Phys. (N.Y.) 245, 339 (1996) ; Mod. Phys. Lett. A 11, 397 (1996).

16. N. Fleury and M. Rausch de Traubenberg, Mod. Phys. Lett. A 11, 899 (1996) ; M. Rausch de Traubenberg and M.J. Slupinski, Mod. Phys. Lett. A 12, 3051 (1997) ; preprint: hep-th/9904126 ; M. Rausch de Traubenberg, preprint: hep-th/0007150.

17. A. Mostafazadeh, Int. J. Mod. Phys. A 11, 2957 (1996).

18. M. Daoud and Y. Hassouni, Prog. Theor. Phys. 97, 1033 (1997).

19. C. Quesne and N. Vansteenkiste, Phys. Lett. A 240, 21 (1998).

20. M. Daoud, Y. Hassouni and M. Kibler, The $k$-fermions as objects interpolating between fermions and bosons, in Symmetries in Science $X$, eds. B. Gruber and M. Ramek (Plenum Press, New York, 1998), pp. 63-77 ; Yad. Fiz. 61, 1935 (1998).

21. M. Kibler and M. Daoud, An alternative basis for the Wigner-Racah algebra of the group SU(2), to be published in Turkish J. Phys. (2000) ; 
preprint: physics/9712034.

22. M. Daoud and M. Kibler, A fractional supersymmetric oscillator and its coherent states, to be published in Turkish J. Phys. (2000) ; preprint: math-ph/9912024.

23. H. Ahmedov and Ö.F. Dayi, preprint: math.QA/9903093.

24. R.S. Dunne, A.J. Macfarlane, J.A. de Azcárraga and J.C. Pérez Bueno, Phys. Lett. B 387, 294 (1996) ; Czech. J. Phys. 46, 1145 (1996) ; 46, 1235 (1996).

25. M. Mansour, M. Daoud and Y. Hassouni, Phys. Lett. B 454, 281 (1999).

26. M. Arik and D.D. Coon, J. Math. Phys. 17, 524 (1976). A.J. Macfarlane, J. Phys. A: Math. Gen. 22, 4581 (1989). L.C. Biedenharn, J. Phys. A: Math. Gen. 22, L873 (1989). C.-P. Sun and H.-C. Fu, J. Phys. A: Math. Gen. 22, L983 (1989). A.I. Solomon, Phys. Lett. A 196, 29 (1994).

27. M. Chaichian, D. Ellinas and P.P. Kulish, Phys. Rev. Lett. 65, 980 (1990). J. Katriel and A.I. Solomon, J. Phys. A: Math. Gen. 24, 2093 (1991). R.J. McDermott and A.I. Solomon, J. Phys. A: Math. Gen. 27, L15 (1994) ; 27, 2037 (1994). V.I. Man'ko, G. Marmo, E.C.G. Sudarshan, F. Zaccaria, Phys. Scripta 55, 520 (1997). F.B. Wang and L.M. Kuang, Phys. Lett. A 169, 225 (1992). L.M. Kuang and F.B. Wang, Phys. Lett. A 173, 221 (1993). Le-Man Kuang, Fa-Bo Wang and Gao-Jian Zeng, Phys. Lett. A 176, 1 (1993).

28. G.A. Goldin, R. Menikoff and D.H. Sharp, J. Math. Phys. 21, 650 (1980) ; 22, 1664 (1981) ; G.A. Goldin and D.H. Sharp, Phys. Rev. Lett. 76, 1183 (1996).

29. J.M. Leinaas and J. Myrheim, Nuovo Cimento B 37, 1 (1977).

30. A. Le Clair and C. Vafa, Nucl. Phys. B 401, 413 (1993). See also: C. Ahn, D. Bernard and A. Le Clair, Nucl. Phys. B 346, 409 (1990).

31. S. Majid and M.J. Rodríguez-Plaza, J. Math. Phys. 35, 3753 (1994).

32. A. Vourdas, Phys. Rev. A 41, 1653 (1990) ; 43, 1564 (1991) ; A. Vourdas and C. Bendjaballah, Phys. Rev. A 47, 3523 (1993) ; A. Vourdas, J. Phys. A: Math. Gen. 29, 4275 (1996).

33. Y. Bérubé-Lauzière and V. Hussin, J. Phys. A: Math. Gen. 26, 6271 (1993).

34. A. Metz, J. Jolie, G. Graw, R. Hertenberger, J. Gröger, C. Günther, N. Warr and Y. Eisermann, Phys. Rev. Lett. 83, 1542 (1999). See also: F. Iachello, Nucl. Phys. News 10, 12 (2000). 